\title{
Modeling and Proposal of a Black Phosphorus- based Nanostructure for Detection of Avian Influenza Virus in THz Region
}

Elaheh Hoseini

Lorestan University

Ali Mir ( $\square$ mir.a@lu.ac.ir)

Lorestan University https://orcid.org/0000-0001-7196-6513

Ali Farmani

Lorestan University

\section{Research Article}

Keywords: Black Phosphorus, Nanostructure, Grating, Surface Plasmon, Refractive Index, Virus detection.

Posted Date: October 20th, 2021

DOI: https://doi.org/10.21203/rs.3.rs-897041/v1

License: (c) (i) This work is licensed under a Creative Commons Attribution 4.0 International License.

Read Full License 


\title{
Modeling and proposal of a Black Phosphorus-based Nanostructure for Detection of Avian Influenza Virus in THz Region
}

\author{
Elahe Hoseini ${ }^{1}$, Ali Mir $^{* 1}$, and Ali Farmani ${ }^{1}$ \\ ${ }^{1}$ Department of Electrical Engineering, Lorestan University, Khorramabad, Iran \\ *Corresponding author e-mail: mir.a@lu.ac.ir
}

\begin{abstract}
In this paper, a multilayer/monolayer black phosphorus (BP)-based nanostructure is presented to detect the avian influenza virus. The nanostructur is a grating arrangement made of $\mathrm{BP}$ over $\mathrm{SiO}_{2}$ or $\mathrm{Al}_{2} \mathrm{O}_{3}$ substrate. To achieve the transmission spectrum, depend on the changes in the lateral length of BP, namely ( $\mathrm{L}=100,150,170 \mathrm{~nm})$ as well as the complex refractive index of each of three viruses types (H1N1, H5N2, H9N2) in the THz range, the structure is numerically simulated by 3D Finite Difference Time Domain (3D-FDTD) method. The change in resonance frequency is greater for the H9N2 virus because the real part of its refractive index is relatively larger. Here, too, the rate of change is examined based on the different thicknesses of the H9N2 virus. Also, changes in the refractive index of the environment have been used to calculate important parameters in the sensors, such as sensitivity, FWHM, and figure of merit. Overall, this platform provides a promising platform for detecting influenza viruses.
\end{abstract}

KEYWORDS: Black Phosphorus; Nanostructure; Grating; Surface Plasmon; Refractive Index; Virus detection.

\section{INTRODUCTION}

Nowadays, numerous applications such as health care, disease detection, food quality control, and the ability to quickly identify biomolecules make biosensors one of the most research fields in recent years [1]. One of the detection techniques in biosensors is free-label methods. Free-label methods have advantages such as reliability, high sensitivity, cheapness, ease of integration, and low damage to analytes. Therefore, provides them as an excellent candidate for simple and fast devices [2]. Many optical detection principles have been investigated, like photonic crystals [3], optical waveguides [4], fiber optics [5], resonant mirrors [6], surface plasmon resonance (SPR) [7], and Mach-Zehnder [8]. Biosensors can be classified into different kinds according to different signal recognition mechanisms such as resonant, electrochemical, ion-sensitive field-effect transistors (ISFETs), thermal detection, and photometric sensors. The resonant biosensor is usually employed to detect biomolecules in aquatic environments. These types of sensors depend on the viscosity, change of mass, and conductivity. Numerous biosensors like a surface acoustic wave (SAW) and magnetoelastic sensors, quartz crystal (piezoelectric sensors) biosensors fall into this class [9]. Electrochemical techniques are a good choice for this type of technology due to their simplicity, high sensitivity, and desirable properties. This type of sensor is one of the biological reactions that result from ionic production and consumption. This will reason the charge transfer through the two layers of the physio-chemical converter which produces the measurable signal. These types of biosensors have three major categorizations of such as potentiometric, amperometry, and impedimetric silicon-based chips [10-12]. ISFETs are usually developed to measure $\mathrm{pH}$ and ion concentration [13-15]. New thermal biosensors have been developed due to enzymatic conversion to control enzymatic reactions so that most biochemical reactions have exothermic properties [16]. In photometric sensors, the output signal is measured in light intensity. So, a measurement system provides a simple, fast, repeatable, and labelfree test for fixed analytes. SPR-based sensors have been attracted a lot of attention due to their good advantages of small size and high sensitivity [17-18]. Each biosensor has several important parameters including, sensitivity, selectivity, dynamic range, response time, repeatability, lifetime, stability characteristic, low production cost, low equipment, small footprint, low power consumption, suitable operating temperature [19]. One of the important features in the field of plasmonic is the reduction of photonic devices.

Therefore, the use of two-dimensional (2D) materials with similar layered structures in the field of high performance and flexibility of nanoelectronics and photonics is on the agenda of researchers. Two-dimensional materials like graphene, transition metal dichalcogenides (TMDs), and black phosphorus (BP) with atomic thickness have been attracting the attention of many researchers in recent years [20-22].

Over the past decade, the advent of graphene has led to extensive research into other 2D materials. Graphene, as a 2D semiconductor, has a bandgap of zero or close to zero and a slight absorption coefficient that limits its usages in 
situations where a high on-off current ratio and intense light-material reactions are needed [23-26]. In contrast, TMDs such as $\mathrm{MoS}_{2}, \mathrm{MoSe}_{2}$, and $\mathrm{MoTe}_{2}$ have a limited bandgap of 1 to $2 \mathrm{ev}$, they have a high on-off current ratio, however, the narrow range of the bandgap and their low mobility may prevent their use in many fields (Such as FETs and IR optoelectronics) [27]. One another 2D material is BP that shows a direct and adjustable bandgap that depends on the number of layers (For example, bulk $0.3 \mathrm{ev}$, two layers $1.88 \mathrm{ev}$, single layer $2 \mathrm{ev}$ ) [28]. Thus, single-layer BP as an option for bandwidth optical applications the infrared and terahertz spectra are considered [27]. Therefore, as a plasmonic platform, it enables atomic-scale thin BP to confine plasmons in a very small amplitude and increase lightmater reactions [29]. Due to the mobility carrier $1000 \mathrm{~cm}^{2} \mathrm{~V}^{-1} \mathrm{~s}^{-1}$ and the bandgap of $0.3 \mathrm{ev}$, BP can be used as a suitable option for the design of absorbers [30]. 2D BP has strong anisotropic electrical and optical properties in the plane. Both single and multi-layer BP shapes make it an ideal semiconductor device for various applications, like efficient photo-electrical conversion and ultra-light emission, due to its adjustable direct bandgap. As the number of layers increases, the interactions within the layers are determined. Single-layer / multi-layer BP has a high bandgap value compared to its balk shape. The basic optical properties in anisotropic light absorption such as their narrowdirected bandgap, excitation effect, photoconductivity is usually determined by measuring their linear absorption, while under adjustable optical properties, the desired feature for their photonic devices and semiconductors are considered [31].

In this regard, researchers have proposed many structures based on BP. In 2018, Paul et al. proposed a BP-based biosensor to detect DNA hybridization that exhibits $125^{\circ} / \mathrm{RIU}$ sensitivity, 0.95 detection accuracy, and $13.62 \mathrm{RIU}^{-1}$ quality factor [32]. In 2019, a gas sensor structure was proposed by Singh et al. to increase the surface plasmon resonance sensitivity [33]. In the same year, a BP-JL-RC-MOSFET biosensor was introduced by Kumar et al. for electrical detection of biomolecules of deadly diseases [34]. For the ultrasensitive detection of human neuron-specific enolase cancer biomarkers, the first BP-fiber-optic biosensor was proposed by Zhou et al. in 2019 [35]. Jia et al. presented a highly sensitive plasmon biosensor through vertically stacking halloysite nanotubes (HNTs). The HNTs$\mathrm{MoS}_{2}$ composite layer dramatically increases the long-term stability of BP as well as the angular and phase-detection sensitivities due to its thickness of several hundred nanometers [36]. Recently BP-TMDC based SPR biosensor was investigated by Sarika et al. the sensitivity of the proposed biosensor with Si, BP with TMDCs is better than the results achieved for Si-graphene and Si-TMDCs based SPR biosensor [37].

Despite the excellent applications of BP in the above articles, but three types of Influenza viruses, H1N1, H5N2, and H9N2, have been less studied by research groups. One of the main pathogens between humans and other species that cause seasonal epidemics each year is the Influenza A Virus (IAV). H1N1 is one of the subtypes of IAV, the influenza virus H1N1, generally referred to as the "swine flu", in April 2009 spread rapidly around the world. Influenza virus infection occurs by inhaling particles that contain the virus or through contact with infected surfaces, and respiratory infection is characterized via acute symptoms like high fever, lethargy, and cough [38]. Influenza viruses of the H9N2 subset have appeared in various species of poultry in Eurasia and Africa, causing periodic infections in humans and mammals. China can be considered the main center of the H9N2 virus epidemic [39]. H5N2 Influenza virus activity sometimes has been seen in Taiwanese chickens in the last few years. The first outbreak of low-pathogenic avian influenza (LPAI) H5N2 viruses in late 2003 and the second outbreak of LPAI in 2008 was observed. Genetic analysis of the Taiwanese H5N2 viruses shows that their surface protein genes, hemagglutinin (HA) and neuraminidase (NA), were not taken from the source viruses of the Eurasian gene but were closely related during the 1994 outbreak to the H5N2 virus isolated from chicken in Mexico [40]. Therefore, the purpose of this paper is to introduce a biosensor based on the valuable properties of BP for the detection of $\mathrm{H} 1 \mathrm{~N} 1, \mathrm{H} 5 \mathrm{~N} 2$, and H9N2 viruses.

\section{THEORY AND ANALYTICAL TREATMENT}

Fig. 1(a) presents the BP nanostructure as a square array of $\mathrm{BP}$ as the main cell with a period of $\mathrm{P}=200 \mathrm{~nm}$ to measure the transmission through a biosensor chip for viruses detection. The square $\mathrm{BP}$ array is covered on the $\mathrm{SiO} 2$ or $\mathrm{Al}_{2} \mathrm{O}_{3}$ substrate. Multilayer/monolayer BP nano-flake has dimensions $\mathrm{L} \times \mathrm{L} \times \mathrm{d}$ with $\mathrm{L}=100,150,170 \mathrm{~nm}$, and d = $2 \mathrm{~nm}$, and the distance between the two layers of BP is $1 \mathrm{~nm}$. The top cover viruses is a layer of air. 

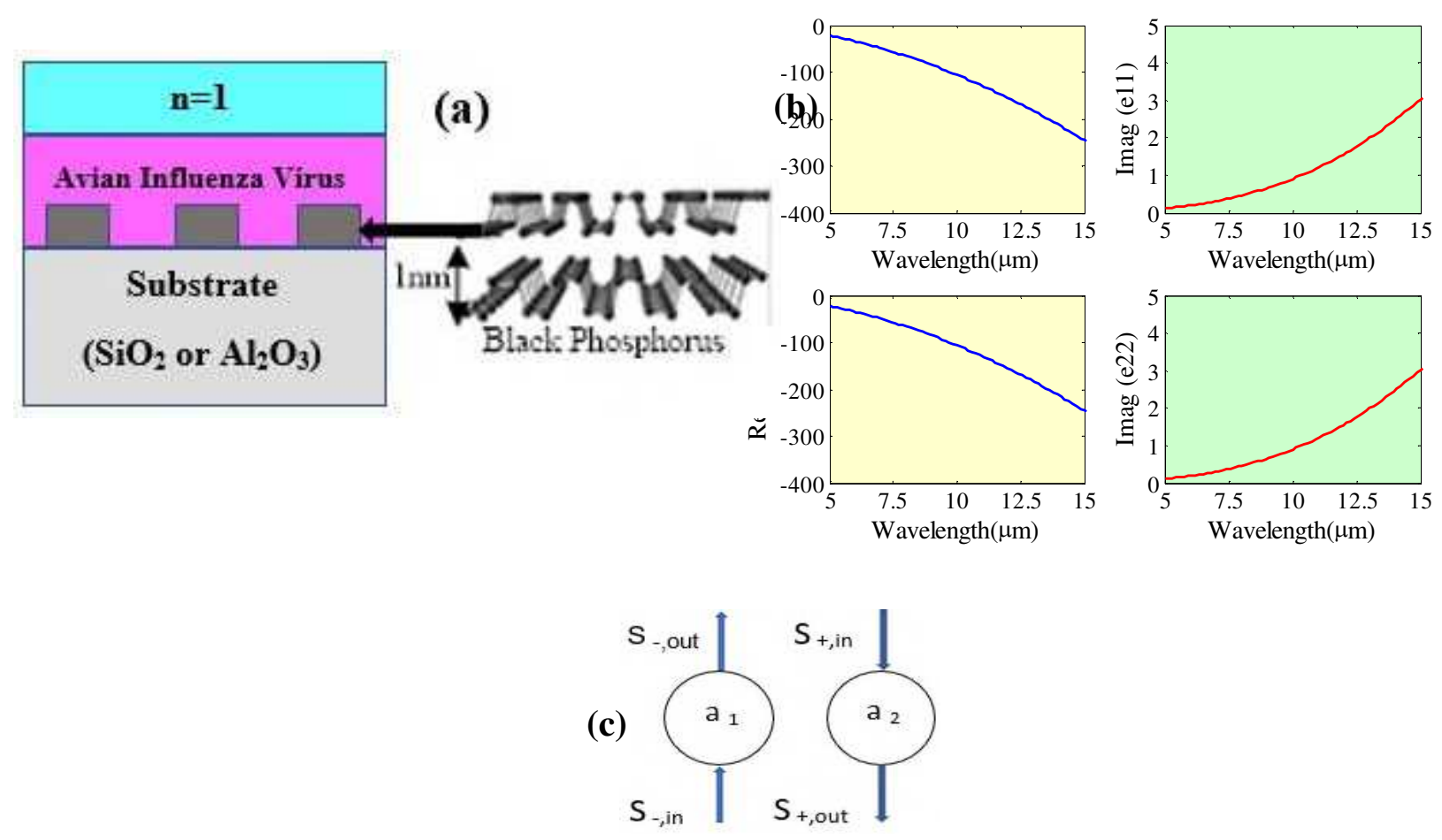

Fig. 1. (a) the proposed nanostructure BP nanoflake for Avian Influenza viruses detection, (b) The real and imaginary parts of the relative permittivity tensor component $\varepsilon_{11}$ and $\varepsilon_{22}$, respectively, (c) The CMT model with two resonant modes of the nanostructured BP system.

According to the optical properties of BP, using the Drude model, the conductivity of few-layer BP can be defined as follows [41]:

$$
\sigma_{j j}=\frac{i D_{j}}{\pi\left(\omega+\frac{i \eta_{e}}{\mathrm{~h}}\right)}
$$

where $j(=1,2)$ indicates the direction of $\mathrm{x}$ or $\mathrm{y}$, and $D j$ is the weight of Drude model along the $j$-axis, equal to $\pi \mathrm{e}^{2} \mathrm{n}_{\mathrm{sj}} / \mathrm{m}_{\mathrm{j}}$. Also, $\eta_{e}$ and $\omega$ represent the scattering rate and frequency of the incoming light, respectively. Finally, $\mathrm{m} 1$ and $\mathrm{m} 2$ are the electron effective masses near the $\Gamma$ point which can be written as follows using the Hamilton model.

$$
m_{1}=\mathrm{h}^{2} /\left(2 \gamma^{2} / \Delta+\eta_{c}\right), m_{2}=\mathrm{h}^{2} / v_{c}
$$

The electronic density of BP, $n_{s}$, with thickness of $d$ is calculated as [30]:

$$
n_{s}=\left(\begin{array}{cc}
n_{s 1} & 0 \\
0 & n_{s 2}
\end{array}\right)=\left(\begin{array}{cc}
m_{1} & 0 \\
0 & m_{2}
\end{array}\right) \frac{k_{B} T}{d \pi \mathrm{h}^{2}} \ln \left[1+\exp \left(\frac{E_{f}-E_{c}}{k_{B} T}\right)\right]
$$

where $\mathrm{k}_{\mathrm{B}}$ is the Boltzmann constant, $\mathrm{T}$ is temperature, and $E_{f}-E_{c}$ is the relative Fermi energy level. Using a layer of phosphorene with volumetric anisotropic permittivity, 2D surface conductivity can be expressed as 3D surface conductivity through the relation of $\sigma_{2 \mathrm{D}}=\mathrm{d} \times \sigma_{3 \mathrm{D}}$. This allows the relative permittivity diagonal tensor $\varepsilon_{\mathrm{D}}\left(\varepsilon_{11}, \varepsilon_{22}\right.$, $\left.\varepsilon_{33}\right)$ to be defined for BP with thickness of $d$. The permittivity of a 2D-BP can be calculated by [42, 30]:

$$
\varepsilon_{j j}=\varepsilon_{r}+i \frac{\sigma_{j j}}{\varepsilon_{0} \omega d}=\varepsilon_{r}-\frac{\varepsilon^{2} n_{s j}}{\varepsilon_{0} m_{j}} \frac{1}{\omega\left(\omega+i \gamma_{b p}\right)}
$$

The value of the BP collision frequency, $\gamma_{\mathrm{bp}}$ of Drude model in the infrared range is approximately equal to $\eta_{\mathrm{e}} / \hbar$, and the $\mathrm{z}$ component is $\varepsilon_{33}=\varepsilon_{\mathrm{r}}=5.76$, for $2 \mathrm{D}-\mathrm{BP}$. The real and imaginary parts of the relative permittivity components are shown in Fig. 1(b). According to it, the real part of the permittivity components at the wavelength of $5 \mu \mathrm{m}$ has a greater value than at the wavelength of $15 \mu \mathrm{m}$. In the imaginary part, on the other hand, the components of the relative permittivity act inversely to the real part. The properties of BP surface plasmon in nanostructure BP can be expressed by the coupled-mode theory (CMT) method according to Fig. 1(c). Also, the incoming and outgoing light waves $\mathrm{S}_{ \pm, \text {in(out) }}$ are shown in Fig. 1(c). When the incoming light wave $\mathrm{S}_{+, \text {in }}$ passes through the BP grating, according to the SPR effect the energy can be coupled to the BP grating. BP surface plasmon resonance modes are inspired by the BP 
grating region $\mathrm{a}_{\mathrm{m}}(\mathrm{m}=1,2)$ due to the energy range, where $\mathrm{a}_{\mathrm{m}}$ is the $\mathrm{m}^{\text {th }}$ mode of the resonance modes with resonant frequency $\omega_{\mathrm{m}}$. To calculate the absorption coefficient, we first need to calculate the transmission and reflection coefficients, $t\left(\omega, E_{f}\right)=\mathrm{S}_{+, \text {out }} / \mathrm{S}_{+, \text {in }}$ and $\mathrm{r}\left(\omega, E_{f}\right)=\mathrm{S}_{-, \text {out }} / \mathrm{S}_{+, \text {in }}$ of BP gratings plasmonic, respectively, that can be obtained from the following equations [30]:

$$
\begin{aligned}
& t(\omega)=1+\left(\frac{\gamma_{1}}{\tau_{\omega 2}}+\frac{\gamma_{2}}{\tau_{\omega 1}}+\chi_{2} \sqrt{\frac{1}{\tau_{\omega 2}} \frac{1}{\tau_{\omega 1}}}+\chi_{1} \sqrt{\frac{1}{\tau_{\omega 2}} \frac{1}{\tau_{\omega 1}}}\right) /\left(\gamma_{1} \gamma_{2}-\chi_{1} \chi_{2}\right) \\
& r(\omega)=\left(\frac{\gamma_{1}}{\tau_{\omega 2}}+\frac{\gamma_{2}}{\tau_{\omega 1}}+\chi_{2} \sqrt{\frac{1}{\tau_{\omega 2}} \frac{1}{\tau_{\omega 1}}}+\chi_{1} \sqrt{\frac{1}{\tau_{\omega 2}} \frac{1}{\tau_{\omega 1}}}\right) /\left(\gamma_{1} \gamma_{2}-\chi_{1} \chi_{2}\right)
\end{aligned}
$$

As a result, the absorption efficiency in the light spectrum is defined as follows:

$$
A(\omega)=1-|t(\omega)|^{2}-|r(\omega)|^{2}
$$

$1 / \tau_{\omega \mathrm{m}}$ and $\tau_{\omega \mathrm{m}}$ show the decay rate and the lifetime, respectively, due to the energy coupling of each mode in the light field. Also, $1 / \tau_{\mathrm{im}}$ and $\tau_{\mathrm{im}}$ determine the decay rate and lifetime, respectively, due to inherent losses of $\mathrm{m}^{\text {th }}$ mode in $\mathrm{BP}$ grating. The coupling coefficient between two resonance modes is $\mu_{\mathrm{pq}}$ which can be considered as $\chi_{1}=i \mu_{12}$ and $\chi_{2}=\mathrm{i} \mu_{21}$. On the other hand, $\chi_{1}, \chi_{2}, \tau_{m}, \tau_{i m}$ and $\omega_{m}$ are used to describe the absorption coefficient of BP absorption equation, $\mathrm{A}(\omega)$.

For identifying viruses, each of these three types of viruses has a complex refractive index of $N=\alpha n+i \beta \kappa$, while $\alpha$ and $\beta$ are the frequency-independent parameters in the range of 1-1.4 for $n$ and 1-2 for $\kappa$ and also identify the different types of viruses and protein concentrations. Finally, $n$ and $\kappa$ are the real and imaginary parts of the dielectric constant that can be extracted using the following relations [43].

$$
\begin{aligned}
& \sqrt{\varepsilon(\omega)}=\eta \eta(\omega)=n(\omega)+i \kappa(\omega) \\
& \varepsilon(\omega)=1.5^{2}-\frac{\omega_{p}^{2}}{\omega^{2}-\omega_{0}^{2}+i \omega \gamma}
\end{aligned}
$$

where $\omega_{p}$ and $\gamma$ have a same value, equal to $4 \mathrm{THz}$.

\section{RESULTS AND DISCUSION}

The three-dimensional Finite Difference Time Domain (3D-FDTD) method is used in our simulations with the perfectly matched absorbing boundary conditions for the below and top of the computational space along the $\mathrm{z}$ direction, and the periodic boundary conditions for the both $\mathrm{x}$ and $\mathrm{y}$-directions. A single unit cell is considered in our 3D simulations. According to Table 1, the total protein concentration and complex refractive index of three Avian Influenza virus samples are measured by advanced biochemical measurements. The three Avian Influenza virus subtypes are H5N2, H1N1, and H9N2.

Table 1. AI virus subtypes, total protein concentration, and Complex refractive
\begin{tabular}{|c|c|c|}
\hline Virus subtype & $\begin{array}{c}\text { Total protein } \\
\text { concentration } \\
(\mathrm{mg} / \mathrm{mL})\end{array}$ & $\begin{array}{c}\text { Relative } \\
\text { Complex refractive } \\
\text { index }\end{array}$ \\
\hline $\mathrm{H} 5 \mathrm{~N} 2$ & 0.2 & $\mathrm{~N}=\mathrm{n}+\mathrm{i \kappa}$ \\
\hline $\mathrm{H} 1 \mathrm{~N} 1$ & 0.54 & $\mathrm{~N}=\mathrm{n}+1.4 \mathrm{i \kappa}$ \\
\hline $\mathrm{H} 9 \mathrm{~N} 2$ & 0.28 & $\mathrm{~N}=1.2 \mathrm{n}+1.4 \mathrm{i \kappa}$ \\
\hline
\end{tabular}

When the light is coupled vertically to the nanostructure BP-based, a propagating wave is generated in the BP flake with an effective wavelength, corresponds to the grating period. If the wavelength of the incoming light is proportional to the grating period, the surface plasmon resonance can be stimulated. To investigate the optical properties of the square array of the $\mathrm{BP}$ flake, the light transmission and absorption spectrums for the proposed nanostructure with two different $\mathrm{SiO}_{2}$ and $\mathrm{Al}_{2} \mathrm{O}_{3}$ substrate, when the $\mathrm{BP}$ length, $\mathrm{L}$, is equal to $150 \mathrm{~nm}$ are plotted in Fig. 2(a) and (b), which have been investigated by the 3D-FDTD simulation method. From these figures, it is clear that there are two resonance frequencies, in which the power transmission is decreased dramatically. In other words, in these two wavelengths, surface plasmons are created and incident power is absorbed by the surface plasmons. When the substrate layer is $\mathrm{SiO}_{2}$, the two surface plasmons are created at about $4.5 \mu \mathrm{m}$ and $9 \mu \mathrm{m}$ respectively. For the $\mathrm{Al}_{2} \mathrm{O}_{3}$ substrate, the surface plasmons are created at wavelengths of $5 \mu \mathrm{m}$ and $15 \mu \mathrm{m}$. Any physical change in the 
proposed nanostructure, like variation in the BP length or environment refractive index, can change the wavelength of surface plasmons or intensity of the transmission spectra. Therefore, the proposed device works as a refractive index biosensor which can detect different Avian Influenza viruses (H5N2, H1N1, and H9N2).

(a)

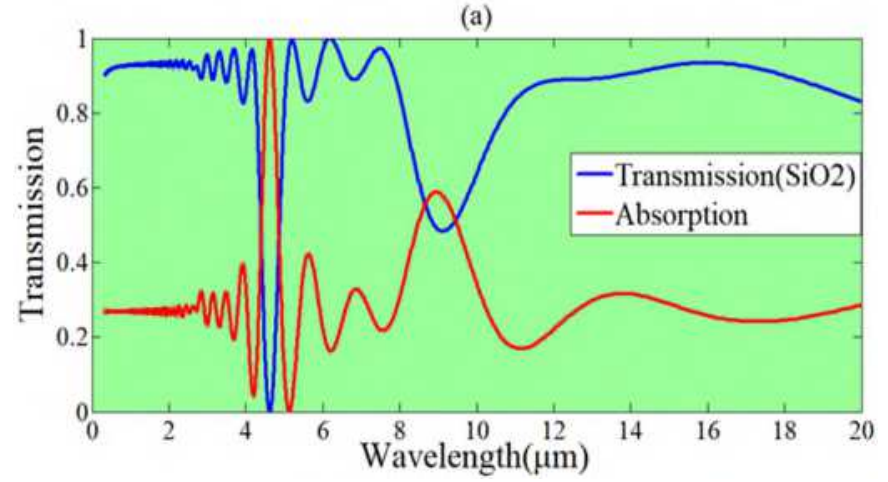

(b)

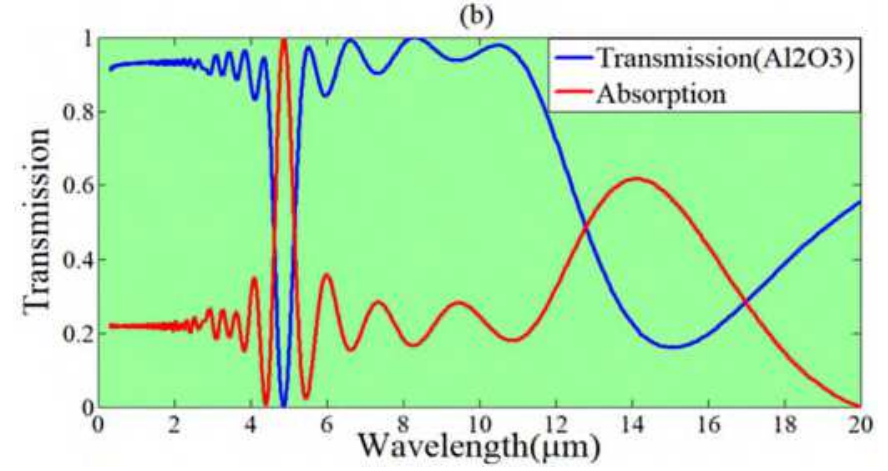

Fig. 2. The simulated results of transmission and absorption spectra at $\mathrm{L}=150 \mathrm{~nm}$ for (a) $\mathrm{SiO}_{2}$ substrate, and (b) $\mathrm{Al}_{2} \mathrm{O}_{3}$ substrate.

Using the L parameter of the proposed structure, the intensity, wavelengths, and amplitudes of the resonance peaks can be adjusted. Therefore, to further investigate the BP surface plasmon in nanostructured, transmission spectra of the two surface plasmon resonance modes with different lengths $(\mathrm{L}=100,150,170 \mathrm{~nm})$ over $\mathrm{SiO}_{2}$ substrate are simulated and presented in Fig. 3. Longer length leads to higher wavelength surface plasmon resonance and larger light absorption value.

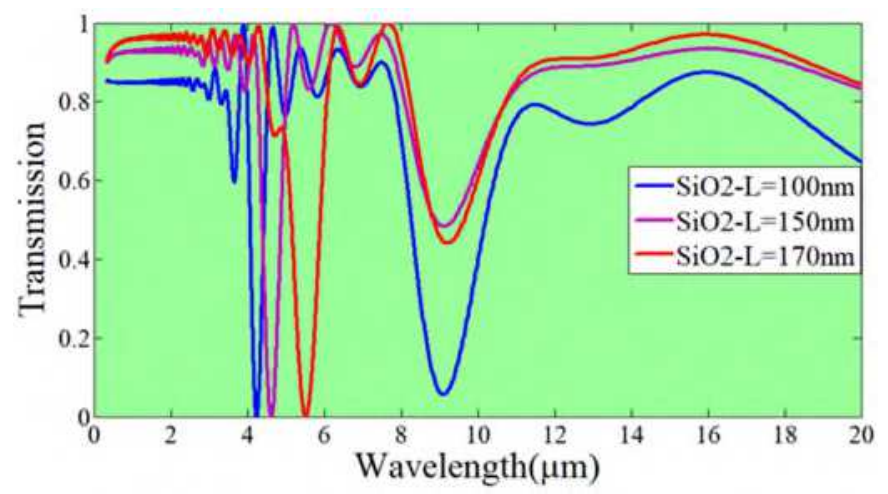

Fig. 3. The simulated results of transmission spectra at $\mathrm{L}=100,150$ and $170 \mathrm{~nm}$ for $\mathrm{SiO}_{2}$ substrate.

In the following section, light transmission spectra with three specific virus samples in the biosensor structure, when the length of $\mathrm{BP}$ equal to $150 \mathrm{~nm}$ with $\mathrm{SiO}_{2}$ and $\mathrm{Al}_{2} \mathrm{O}_{3}$ substrate versus wavelength range from 0.35 to $20 \mu \mathrm{m}$ are examined. The thickness of the desired layer is considered to be $2 \mu \mathrm{m}$ to calculate the transmission spectrum for each of the three virus samples. In this case, the first resonance peaks for $\mathrm{SiO}_{2}$ and $\mathrm{Al}_{2} \mathrm{O}_{3}$ is happened at frequencies of 64.86 THz and 61.538 THz, respectively. Fig. 4(a-b) shows the light transmission spectrum for the three H1N1, $\mathrm{H} 5 \mathrm{~N} 2, \mathrm{H} 9 \mathrm{~N} 2$ virus samples at $\mathrm{L}=150 \mathrm{~nm}$, and the $\mathrm{SiO}_{2}$ and $\mathrm{Al}_{2} \mathrm{O}_{3}$ substrate. As it is clear, the magnitude of transmission for viruses $\mathrm{H} 1 \mathrm{~N} 1$ and $\mathrm{H} 5 \mathrm{~N} 2$ is slightly higher than for virus $\mathrm{H} 9 \mathrm{~N} 2$ and the rate of variation in the resonance frequency for H9N2 virus is higher than that of the two other viruses, H1N1 and H5N2. This can be 
attributed to the fact that the real part of the H9N2 virus refractive index has a larger amount than the two other viruses. Also changing the analyte virus types changed the frequency of surface plasmon resonances.

(a)

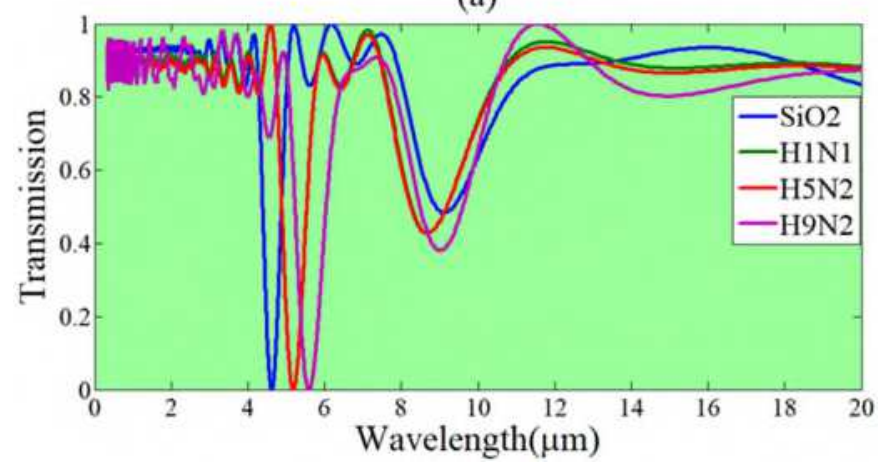

(b)

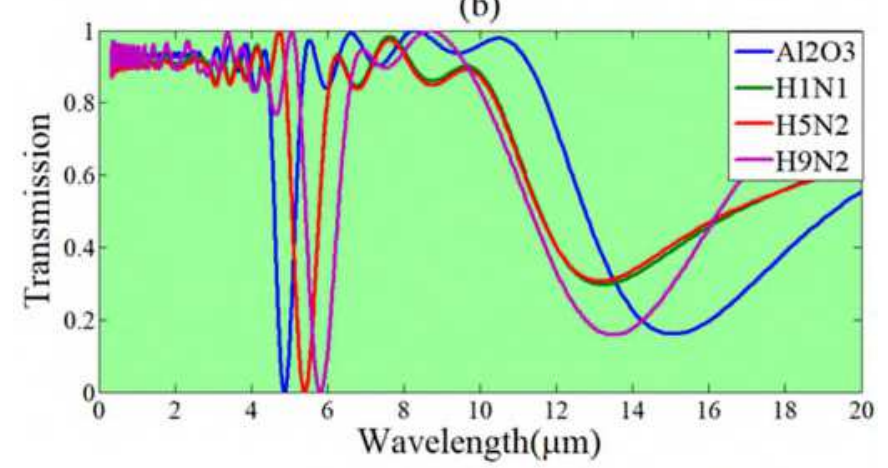

Fig. 4. The simulated results of transmission spectra for different AI virus vs. wavelength when $\mathrm{L}=150 \mathrm{~nm}$ over (a) $\mathrm{SiO}_{2}$ substrate and (b)

$\mathrm{Al}_{2} \mathrm{O}_{3}$ substrate.

In one study, Cheng et al. [43] examined that the size of viruses can be selected from 1 to $8 \mu \mathrm{m}$. Here, we express the volume of the virus by the amount of thickness on the surface of the sensor. In Fig. 5(a-b), for different thicknesses of the H9N2 virus samples from 1 to $4 \mu \mathrm{m}$, the transmission spectrum diagram is shown for $\mathrm{L}=150 \mathrm{~nm}$ over $\mathrm{SiO}_{2}$ and $\mathrm{Al}_{2} \mathrm{O}_{3}$ substrates. As can be seen in the figures, increasing the analyte thickness, increase the amplitude of the transmitted light, so that higher transmission is achieved at higher thicknesses of AI. 
(a)

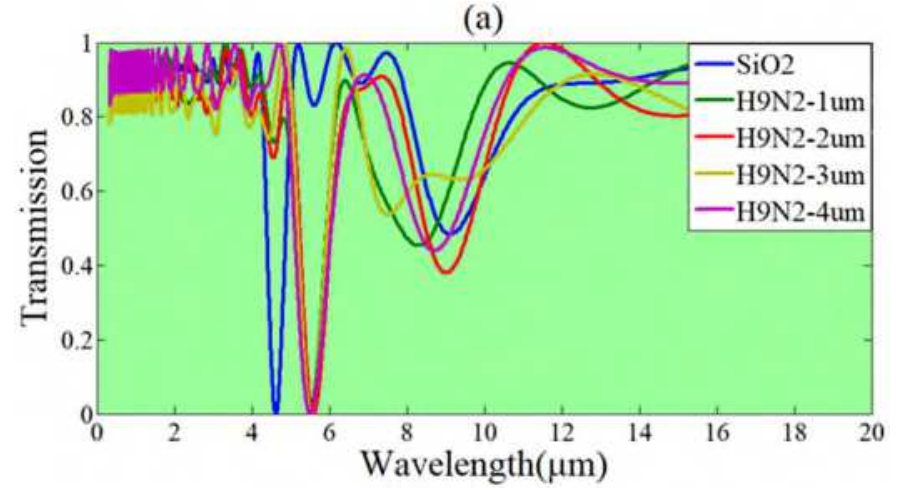

(b)

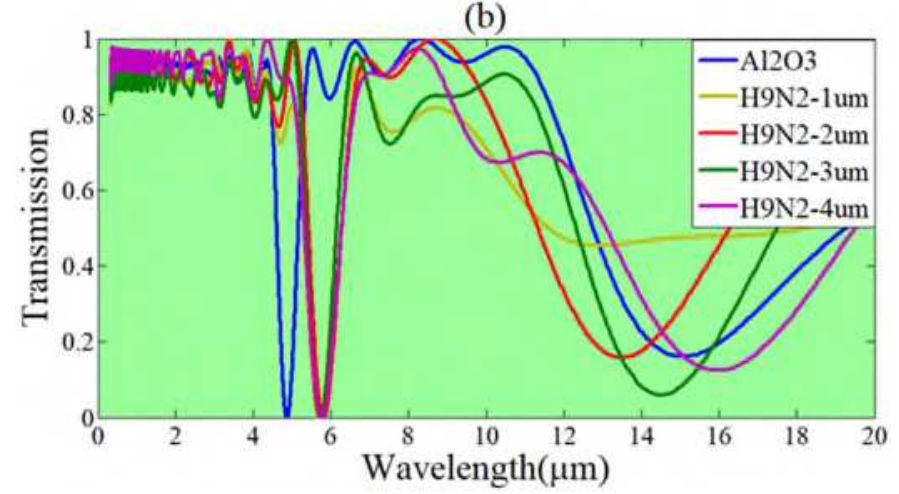

Fig. 5. 3D-FDTD simulation results of transmission spectra for different $\mathrm{H} 9 \mathrm{~N} 2$ analyte thickness when $\mathrm{L}=150 \mathrm{~nm}$ over (a) $\mathrm{SiO}{ }_{2}$ substrate and (b) $\mathrm{Al}_{2} \mathrm{O}_{3}$ substrate.

Refractive index measurement applications are one of the most important applications of BP-based nanostructures. The proposed structure is able to detect the refractive index of its surroundings. This nano-biosensor is very sensitive to the refractive index of the environment. The sensitivity of a biosensor is the ratio of the frequency at resonance peak shift to the change of the refractive index of the environment and is described by the relation [43]:

$$
S=\frac{\Delta \lambda}{\Delta n}
$$

Other important parameters in nanosensors are full width at half maximum (FWHM) and figure of merit $(\mathrm{FoM}=$ $\mathrm{S} / \mathrm{FWHM}$ ). Therefore, in the following section, a table related to the behavior of the proposed structure when $\mathrm{L}$ is equal to $150 \mathrm{~nm}$ in two cases of $\mathrm{SiO}_{2}$ and $\mathrm{Al}_{2} \mathrm{O}_{3}$ substrates, is presented to show the sensitivity, $\mathrm{FWHM}$, and $\mathrm{FoM}$ of the different refractive indexes of the surrounding medium is examined. We first examined the structure using different refractive indexes of the environment. Fig. 6(a-b) show the transmission spectra of the proposed BP-based biosensor for different refractive indexes. It is clear that the surface plasmon resonance wavelength increases as the refractive index increased. According to these graphs, a table related to the sensitivity, FWHM, FOM values is created. Table. 2(a) is for $\mathrm{SiO}_{2}$ substrate and Table. 2(b) is for $\mathrm{Al}_{2} \mathrm{O}_{3}$ substrate. For example, when $\mathrm{n}=1.5$ and substrate is $\mathrm{SiO}_{2}$, the sensitivity, FWHM, and FoM are equal to $9.2 \mathrm{THz} / \mathrm{RIU}, 7.754 \mathrm{THz}$, and 1.186, respectively. If the substrate is $\mathrm{Al}_{2} \mathrm{O}_{3}$, the sensitivity, $\mathrm{FWHM}, \mathrm{FOM}$ in $\mathrm{n}=1.4$ is equal to $7.902 \mathrm{THz} / \mathrm{RIU}, 7.542 \mathrm{THz}, 1.047$, respectively. 
(a)

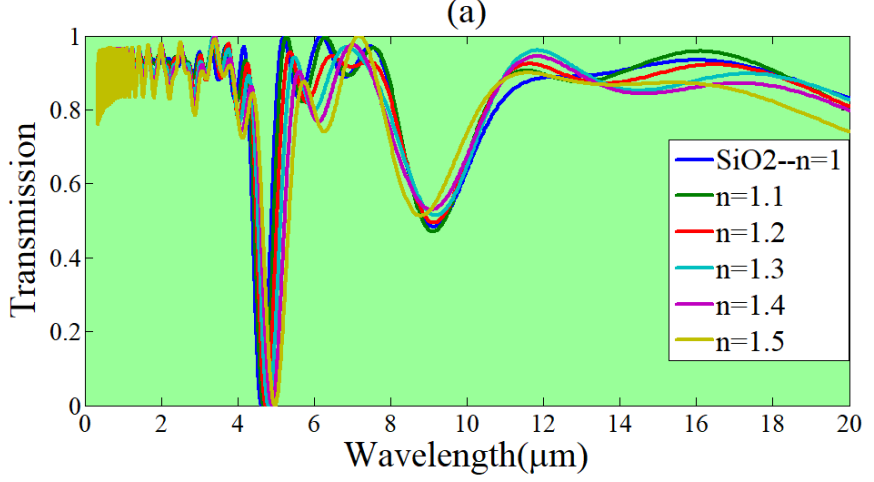

(b)

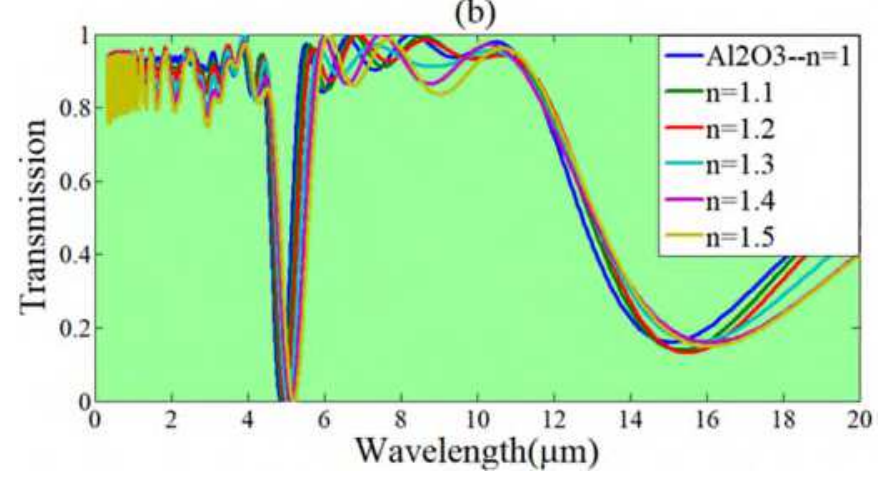

Fig. 6. The simulated transmission spectrum of biosensor in different mediums with $\mathrm{L}=150 \mathrm{~nm}$ for (a): $\mathrm{SiO} 2$ substrate, and (b) $\mathrm{Al}_{2} \mathrm{O}_{3}$ substrate.

Table 2. The Resonance Peak (RP), Sensitivity (S), FWHM and FoM of the proposed structure for (a) $\mathrm{SiO}_{2}$ substrate, and (b): $\mathrm{Al}_{2} \mathrm{O}_{3}$ substrate.

(a)

\begin{tabular}{|c|c|c|c|c|}
\hline $\begin{array}{c}\text { Environment } \\
\text { refractive } \\
\text { Index }\end{array}$ & $\begin{array}{c}\mathrm{RP} \\
{[\mathrm{THz}]}\end{array}$ & $\begin{array}{c}\mathrm{S} \\
{[\mathrm{THz} / \mathrm{RIU}]}\end{array}$ & $\begin{array}{c}\text { FWHM } \\
{[\mathrm{THz}]}\end{array}$ & FOM \\
\hline 1 & 64.86 & --- & 6.48 & --- \\
\hline 1.1 & 64.06 & 8 & 6.665 & 1.2 \\
\hline 1.2 & 63.15 & 8.55 & 6.74 & 1.268 \\
\hline 1.3 & 62.37 & 8.3 & 7.148 & 1.161 \\
\hline 1.4 & 61.224 & 9.09 & 7.27 & 1.25 \\
\hline 1.5 & 60.26 & 9.2 & 7.754 & 1.186 \\
\hline
\end{tabular}

(b)

\begin{tabular}{|c|c|c|c|c|}
\hline $\begin{array}{c}\text { Environment } \\
\text { refractive } \\
\text { Index }\end{array}$ & $\begin{array}{c}\mathrm{RP} \\
{[\mathrm{THz}]}\end{array}$ & $\begin{array}{c}\mathrm{S} \\
{[\mathrm{THz} / \mathrm{RIU}]}\end{array}$ & $\begin{array}{c}\text { FWHM } \\
{[\mathrm{THz}]}\end{array}$ & FOM \\
\hline 1 & 61.538 & --- & 6.631 & --- \\
\hline 1.1 & 60.80 & 7.38 & 6.796 & 1.085 \\
\hline 1.2 & 60.06 & 7.39 & 7.078 & 1.044 \\
\hline 1.3 & 59.323 & 7.383 & 7.165 & 1.030 \\
\hline 1.4 & 58.377 & 7.902 & 7.542 & 1.047 \\
\hline 1.5 & 57.636 & 7.804 & 7.588 & 1.028 \\
\hline
\end{tabular}

In the following, the results are achieved when the BP is monolayer. As can be seen in Fig. 7(a), the light transmission spectra for two different substrates, $\mathrm{SiO}_{2}$ and $\mathrm{Al}_{2} \mathrm{O}_{3}$, has only one surface plasmon resonance, at about $9 \mu \mathrm{m}$ and 15 $\mu \mathrm{m}$ wavelengths, respectively. Also, when the environment refractive index, $\mathrm{n}$, increased, the surface plasmon resonance has a red shift. According to Table. 3(a), for example, the maximum sensitivity, FWHM, and FoM for the case of $\mathrm{SiO}_{2}$ at $\mathrm{n}=1.3$ is equal to $1.06 \mathrm{THz} / \mathrm{RIU}$ and $6.355 \mathrm{THz}, 0.166$, respectively. Despite this, by increasing the value of $\mathrm{n}$ for the case of $\mathrm{Al} 2 \mathrm{O} 3$ substrate, the frequency of resonance peak, increases and causes the creation of a negative sensitivity. 
(a)

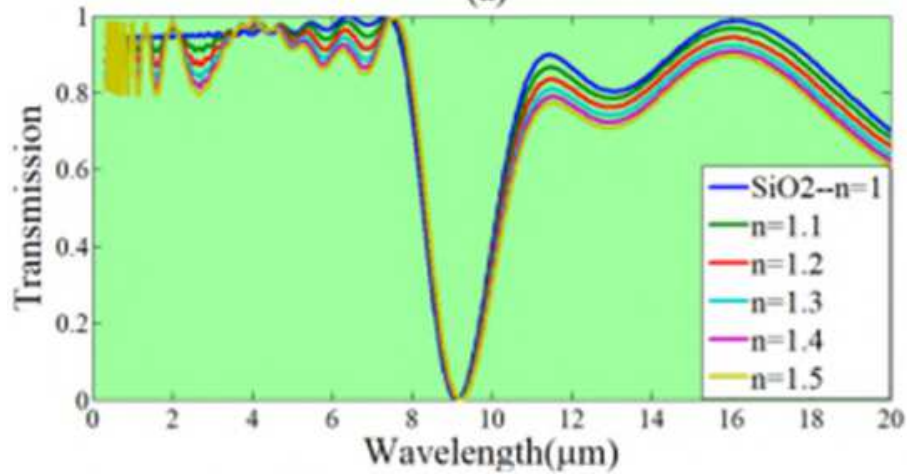

(b)

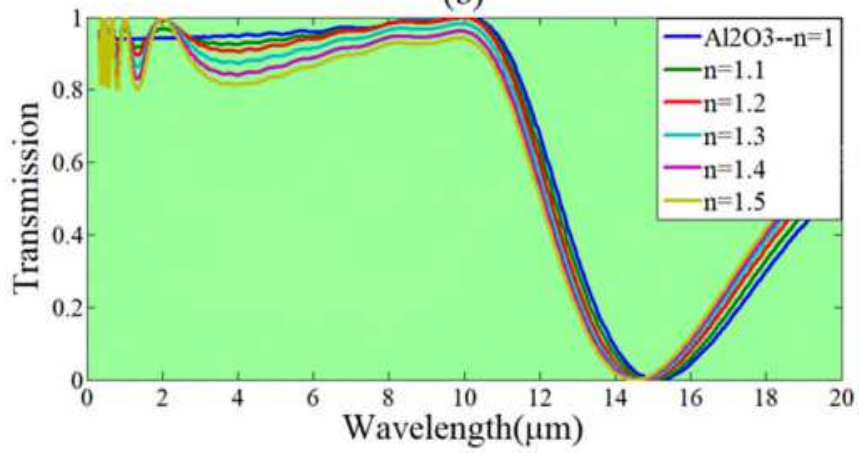

Fig. 7. The simulated transmission spectrum of the proposed biosensor in different mediums for monolayer $\mathrm{BP}$ with $\mathrm{L}=150 \mathrm{~nm}$ for (a): $\mathrm{SiO}{ }_{2}$ substrate, and (b) $\mathrm{Al}_{2} \mathrm{O}_{3}$ substrate.

Table 3. The Resonance Peak (RP), Sensitivity (S), FWHM and FoM of the proposed structure with a monolayer BP for (a) SiO 2 substrate, and (b) $\mathrm{Al}_{2} \mathrm{O}_{3}$ substrate.

(a)

\begin{tabular}{|c|c|c|c|c|}
\hline $\begin{array}{c}\text { Environment } \\
\text { refractive } \\
\text { Index }\end{array}$ & $\begin{array}{c}\text { RP } \\
{[\mathrm{THz}]}\end{array}$ & $\begin{array}{c}\mathrm{S} \\
{[\mathrm{THz} / \mathrm{RIU}]}\end{array}$ & $\begin{array}{c}\text { FWHM } \\
{[\mathrm{THz}]}\end{array}$ & FOM \\
\hline 1 & 32.898 & --- & 6.413 & --- \\
\hline 1.1 & 32.794 & 1.04 & 6.319 & 0.164 \\
\hline 1.2 & 32.686 & 1.06 & 6.328 & 0.167 \\
\hline 1.3 & 32.580 & 1.06 & 6.355 & 0.166 \\
\hline 1.4 & 32.534 & 0.91 & 6.409 & 0.141 \\
\hline 1.5 & 32.478 & 0.84 & 6.428 & 0.130 \\
\hline
\end{tabular}

(b)

\begin{tabular}{|c|c|c|c|c|}
\hline $\begin{array}{c}\text { Environment } \\
\text { refractive } \\
\text { Index }\end{array}$ & $\begin{array}{c}\text { RP } \\
{[\mathrm{THz}]}\end{array}$ & $\begin{array}{c}\mathrm{S} \\
{[\mathrm{THz} / \mathrm{RIU}]}\end{array}$ & $\begin{array}{c}\text { FWHM } \\
{[\mathrm{THz}]}\end{array}$ & FOM \\
\hline 1 & 19.84 & --- & 8.423 & --- \\
\hline 1.1 & 20.05 & -2.1 & 8.375 & -0.250 \\
\hline 1.2 & 20.27 & -2.15 & 8.393 & -0.256 \\
\hline 1.3 & 20.47 & -2.1 & 8.438 & -0.248 \\
\hline 1.4 & 20.57 & -1.825 & 8.414 & -0.216 \\
\hline 1.5 & 21.20 & -2.72 & 8.404 & -0.323 \\
\hline
\end{tabular}




\section{CONCLUSIONS}

In this paper, we propose a BP-based nanostructure (monolayer/multilayer) as a suitable structure for the detection of avian influenza viruses (H1N1, H5N2, H9N2) that have different refractive index values. We observed that the resonance frequency of the nano-biosensor changes for different lengths of $\mathrm{BP}(\mathrm{L}=100,150,170 \mathrm{~nm}) \mathrm{and}_{(\mathrm{SiO}}$ and $\mathrm{Al}_{2} \mathrm{O}_{3}$ ) substrates. By placing the avian influenza viruses, the resonance frequency changes, so we can detect them by considering the variation in the resonance frequency change. The change of the resonance frequency of H9N2 virus is greater, due to the greater amount of the real part of its complex refractive index than the other avian influenza virus subtypes. It can be concluded that the real part of the virus refractive index is related to the resonance frequency and the imaginary part is related to the rate of virus transmission in the spectrum responses. In addition, in the proposed nanostructure, we examined the rate of transmission based on the different thicknesses of H9N2 virus. As the thickness of $\mathrm{H} 9 \mathrm{~N} 2$ virus increases from $1 \mu \mathrm{m}$ to $4 \mu \mathrm{m}$, the amount of transmission increases. We also examined important parameters of the sensors such as sensitivity, FWHM, and FOM. According to these parameters, we can also use of this sensor for refractive index sensing. Overall, introduced BP nanostructure-based THz sensing, can provide a quick solution for the detection of avian influenza viruses by a label-free manner.

\section{REFERENCES}

[1] Mohamad, N., \& Najmeh, N. (2020). Multi-band MIM refractive index biosensor based on Ag-air grating with equivalent circuit and T-matrix methods in near-infrared region. Scientific Reports (Nature Publisher Group), 10(1).

[2] X. Chen, Y. Liu, J. Huang, W. Liu, J. Huang, Y. Zhang, and W. Fu, "LabelFree Techniques for Laboratory Medicine Applications", Frontiers in Laboratory Medicine, vol. 1, pp. 82-85, 2017.

[3] Liu, Y., Wang, H., Ho, J., Ng, R. C., Ng, R. J., Hall-Chen, V. H., ... \& Greer, J. R. (2019). Structural color three-dimensional printing by shrinking photonic crystals. Nature communications, 10(1), 1-8.

[4] Horvath, R. et al. Effect of patterns and inhomogeneities on the surface of waveguides used for optical waveguide lightmode spectroscopy applications. Appl. Phys. B 72,441-447 (2001).

[5] Yin, M. J., Gu, B., An, Q. F., Yang, C., Guan, Y. L., \& Yong, K. T. (2018). Recent development of fiber-optic chemical sensors and biosensors: Mechanisms, materials, micro/nano-fabrications and applications. Coordination Chemistry Reviews, 376, 348-392.

[6] Cush, R. et al. The resonant mirror: a novel optical biosensor for direct sensing of biomolecular interactions. Part 1. Principle of operation and associated instrumentation. Biosens. Bioelectron. 8,347-353 (1993).

[7] Jahanshahi, P., Zalnezhad, E., Devi Sekaran, S. \& Adikan, F. R. M. Rapid Immunoglobulin M-Based Dengue Diagnostic Test Using Surface Plasmon Resonance Biosensor. Sci. Report 4,3851 (2015).

[8] Zinoviev, K. et al. Silicon Photonic Biosensors for Lab-on-a-Chip Applications. Adv. Opt. Technol. 2008,383927 (2008).

[9] Interfacial Structures and Properties of Organic Materials for Biosensors: An Overview

[10] Sethi, R.S.; Lowe, C.R. Electrochemical microbiosensors. In Proceedings of IEE Colloquium on Microsensors, London, UK, 4 April 1990.

[11]Thévenot, D.R.; Toth, K.; Durst, R.A.; Wilson, G.S. Electrochemical biosensors: Recommended definitions and classification. Biosens. Bioelectr. 2001, 16, 121-131.

[12]Mioslav, P.; Petr, S. Electrochemical biosensors-principles and applications. J. Appl. Biomed. 2008, 6, 57-64.

[13]Moss, S.D.; Janata, J.; Johnson, C.C. Potassium ion-sensitive field effect transistor. Anal. Chem. 1975, 47, 2238-2243.

[14]Duroux, P.; Emde, C.; Bauerfeind, P.; Francis, C.; Grisel, A.; Thybaud, L.; Arstrong, D.; Depeursinge, C.; Blum, A.L. The ion sensitive field effect transistor (ISFET) $\mathrm{pH}$ electrode: A new sensor for long term ambulatory $\mathrm{pH}$ monitoring. Gut 1991, 32, 240-245.

[15]Esashi, M.; Matsuo, T. Integrated Micro Multi Ion Sensor Using Field Effect of Semiconductor. IEEE Trans. Biomed. Eng. 1978, BME-25, 184192.

[16]Zheng, Y.-H.; Hua, T.-C.; Sun, D.-W.; Xiao, J.-J.; Xu, F.; Wang, F.-F. Detection of dichlorvos residue by flow injection calorimetric biosensor based on immobilized chicken liver esterase. J. Food Eng. 2006, 74, 24-29.

[17]Homola, J.; Yee, S.; Gauglitz, G. Surface plasmon resonance sensors: Review. Sens. Actuators B Chem. 1999, 54, 3-15.

[18]Wood, R.W. On a remarkable case of uneven distribution of light in a diffraction grating spectrum. Proc. Phys. Soc. Lond. 1902, 18, $269-275$.

[19]Wilson, J. S. (2004). Sensor technology handbook. Elsevier.

[20]M. Xu, T. Liang, M. Shi, and H. Chen, "Graphene-Like Two-Dimensional Materials," Chem. Rev., vol. 113, no. 5, pp. 3766-3798, Jan. 2013.

[21]R. Mas-Ballest' e, C. G' omez-Navarro, J. G' omez-Herrero, and F. Zamora, "2D materials: To graphene and beyond," Nanoscale, vol. 3, no. 1, pp. 20-30, Sep. 2010.

[22]S. Lu et al., "Broadband nonlinear optical response in multi-layer black phosphorus: An emerging infrared and midinfrared optical material," Opt. Exp., vol. 23, no. 9, pp. 11183-11194, May 2015.

[23]K. Geim and K. S. Novoselov, "The rise of graphene," Nat. Mater., vol. 6, pp. 183-91, 2007.

[24]A. H. C. Neto, N. M. R. Peres, K. S. Novoselov, and A. K. Geim, “The electronic properties of graphene,” Rev. Mod. Phys., vol. 81, pp. 109-162, 2009.

[25]M. Jablan, H. Buljan, and M. Soljacic, "Plasmonics in graphene at infrared frequencies," Phys. Rev. B, vol. 80, p. 245435, 2009.

[26]F. J. Garcia de Abajo, "Graphene plasmonics: challenges and opportunities," Acs Photonics, vol. 1, no. 3, pp. 135-152, 2014.

[27]Fo, Q., Pan, L., Chen, X., Xu, Q., Ouyang, C., Zhang, X., ... \& Zhang, W. (2018). Anisotropic plasmonic response of black phosphorus nanostrips in terahertz metamaterials. IEEE Photonics Journal, 10(3), 1-9.

[28]J. R. Choi, K.W. Yong, J.Y. Choi, A. Nilghaz, Y. Lin, J. Xu, X. Lu," Black Phosphorus and its Biomedical Applications", Theranostics, 10051026(2018).

[29]Z. Liu and K. Aydin, “Localized surface plasmons in nanostructured monolayer black phosphorus," Nano Lett., vol. 16, no. 6, pp. 3457-3462, May 2016.

[30]Zhou, R., Peng, J., Yang, S., Liu, D., Xiao, Y., \& Cao, G. (2018). Lifetime and nonlinearity of modulated surface plasmon for black phosphorus sensing application. Nanoscale, 10(39), 18878-18891.

[31]Boddula, R., \& Asiri, A. M. (Eds.). (2019). Black Phosphorus: Synthesis, Properties and Applications. Springer Nature 
[32]S. Pal, A. Verma, J.P. Saini, S. Raikwar, Y.K. Prajapati," Detection of DNA hybridization using graphene-coated black phosphorus surface plasmon resonance sensor ", Materials Science \& Processing, (2018).

[33] Y. Singh, S.K. Raghuwanshi" Sensitivity Enhancement of the Surface Plasmon Resonance Gas Sensor with Black Phosphorus ", Electromagnetic wave sensors, (2019)

[34]A. Kumar, N. Gupta, M. M. Tripathi, R. Chaujar," Analysis of structural parameters on sensitivity of black phosphorus junctionless recessed channel MOSFET for biosensing application", Microsystem Technologies, (2019).

[35]L. Zhou, C. Liu, Z. Sun, H. Mao, L. Zhang, X. Yu, J. Zhao, X. Chen, " Black phosphorus-based fiber optic biosensor for ultrasensitive cancer diagnosis", Biosensors and Bioelectronics, 140-147(2019).

[36]G.Y. Jia, Z.X. Huang, Y.L. Zhang, Z.Q. Hao, Y.L. Tian,"Ultrasensitive plasmonic biosensors based on halloysite nanotubes/MoS2/black phosphorus hybrid architectures", Journal of Materials Chemistry C, (2019).

[37]Pal, S., Verma, A., Saini, J. P., \& Prajapati, Y. K. (2019). Sensitivity enhancement using silicon-black phosphorus-TDMC coated surface plasmon resonance biosensor. IET Optoelectronics, 13(4), 196-201.22

[38]Kumar, R., Nayak, M., Sahoo, G. C., Pandey, K., Sarkar, M. C., Ansari, Y., ... \& Das, P. (2019). Iron oxide nanoparticles based antiviral activity of H1N1 influenza A virus. Journal of Infection and Chemotherapy, 25(5), 325-329.

[39]Li, C., Wang, S., Bing, G., Carter, R. A., Wang, Z., Wang, J., ... \& Wang, Y. (2017). Genetic evolution of influenza H9N2 viruses isolated from various hosts in China from 1994 to 2013. Emerging microbes \& infections, 6(1), 1-11.

[40]Lee, C. C. D., Zhu, H., Huang, P. Y., Peng, L., Chang, Y. C., Yip, C. H., ... \& Smith, D. K. (2014). Emergence and evolution of avian H5N2 influenza viruses in chickens in Taiwan. Journal of virology, 88(10), 5677-5686.

[41]J. Wang and Y. Jiang, Infrared absorber based on sandwiched two-dimensional black phosphorus metamaterials, Opt. Express, 2017, 25(5), 52065216.

[42]Zhu, Y., Tang, B., \& Jiang, C. (2019). Tunable ultra-broadband anisotropic absorbers based on multi-layer black phosphorus ribbons. Applied Physics Express, 12(3), 032009.

[43] Keshavarz, A., \& Vafapour, Z. (2019). Sensing avian influenza viruses using terahertz metamaterial reflector. IEEE Sensors Journal, 19(13), 51615166.

[44]Lee, D. K., Kang, J. H., Kwon, J., Lee, J. S., Lee, S., Woo, D. H., ... \& Seo, M. (2017). Nano metamaterials for ultrasensitive Terahertz biosensing. Scientific reports, 7(1), 1-6. 